\section{MS32-P15 While you were screening...}

Elisa Nauha ${ }^{1}$, Louis Adriaenssens ${ }^{1}$, Nicholas Blagden ${ }^{1}$, Ian Scowen ${ }^{1}$

1. University of Lincoln, Joseph Banks Laboratories, Green Lane, Lincoln, LN6 7DL, UK

email: enauha@lincoln.ac.uk

Screening for crystal forms can be a laborious process where the desired results are sometimes fleeting. During the process new crystal forms which are not relevant to the research question but which are novel, perhaps challenging and of interest to the crystallographer are often found. Sometimes these discoveries lead to a new research direction and sometimes they are forgotten in desk drawers awaiting publication. I will present "undesired" crystal structures acquired while screening for other crystal forms.

Keywords: cocrystal, polymorph, solvate

\section{MS32-P16 The exciting life of a small adenine molecule}

Dubravka Sisak Jung ${ }^{1}$, Tomislav Stolar ${ }^{2}$, Stipe Lukin ${ }^{2}$, Gordan Horvat $^{2}$, Josip Pozar ${ }^{2}$, Graeme M. Day ${ }^{3}$, Mirta Rubcic ${ }^{2}$, Ivana Biljan $^{2}$, Ivan Halasz

1. DECTRIS Ltd., Baden-Daettwil, Switzerland

2. Department of Chemistry, Faculty of Science, University of Zagreb, Zagreb, Croatia

3. School of Chemistry, University of Southampton, Southampton, United Kingdom

4. Ruđer Bošković Institute, Zagreb, Croatia

email: dubravka.sisak@dectris.com

Once known only as a component of DNA, modern chemistry successfully exploits nucleobase adenine for preparation of supramolecular and metallo-organic systems $[1,2]$. Although commercially available as a solid and widely used in synthesis of targeted entities, the characterization of adenine is still very limited, due to tautomersim, inability to grow single crystals and the poor quality of polycrystalline materials [3]. In our attempt to overcome these hindrances we have found out that the problem is far more complex, and that the solution surpasses the simple solid-state characterization. In order to untangle the somewhat conflicting results obtained during the study, a variety of experimental and theoretical techniques had to be used. The characterization started with a commercial adenine, which proved to be a mixture of two polymorphs, one known (I, $P 2$ /c, $\left.Z^{\prime}=2,[3]\right)$ and one unknown (II). In order to solve the structure of polymorph II, a strategy to purify the commercial batch had to be developed first. Subsequent structural analysis, against all odds, showed that the same tautomer as $\mathbf{I}$, but with the symmetry was $F d d 2$ with $Z^{\prime}=1$. According to some authors, this suggests that the new form should be more stable. This seemed to comply with the quantitative analysis of several commercial batches, where most showed presence of polymorph II in excess. However, a series of experiments on polymorph occurrence conditions, interconversion and thermodynamic relationship recognized polymorph I with $Z^{\prime}=2$ as the more stable form at room temperature, while polymorph II is favored at higher temperatures. Extensive theoretical calculations not only supported these experimental results, but also gave an insight into fine structural details that contribute to stability of a certain form. Although this study covered a good share of solid-state characterization, there are still questions open. Disordered structures and possible new polymorphs are a few that will be discussed to illustrate that isolation and identification of polymorphs continues to be a vital area of research in solid-state chemistry [4,5].

[1] Etter, M. C., Reutzel, S. M., Choo, C. G. (1993) J. Am. Chem. Soc. 115, 4411. [2] Stylianou, K. C. et al. (2011) Chem. Commun. 47, 3389. [3] Mahapatra, S. et al. (2008) Cryst. Growth Des. 8, 1223. [4] Bernstein, J. (2011) Cryst. Growth Des. 11, 632. [5] Bučar, D.-K., Lancaster, R. W., Bernstein, J. (2015) Angew. Chem. Int. Ed. 54, 6972.

Keywords: adenine, polymorphism, structure determination, powder 